\title{
Activités
}

6-1 | avril 2009

Varia

\section{Des références pour des pratiques de formation}

Étude d'une situation de formation technologique supérieure initiale

References for training practices. A study of the situation of initial higher technological training

Referencias pasa prácticas de formación. Estudio de una situación de formación tecnológica superior inicial

Raquel Becerril Ortega, Bernard Calmettes, Bernard Fraysse et Pierre Lagarrigue

(2) OpenEdition

Journals

Édition électronique

URL : http://journals.openedition.org/activites/2101

DOI : 10.4000/activites. 2101

ISSN : 1765-2723

Éditeur

ARPACT - Association Recherches et Pratiques sur les ACTivités

Référence électronique

Raquel Becerril Ortega, Bernard Calmettes, Bernard Fraysse et Pierre Lagarrigue, « Des références pour des pratiques de formation », Activités [En ligne], 6-1 | avril 2009, mis en ligne le 15 avril 2009, consulté le 01 mai 2019. URL : http://journals.openedition.org/activites/2101 ; DOI : 10.4000/ activites. 2101

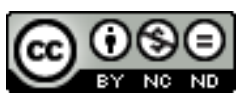

Activités est mis à disposition selon les termes de la licence Creative Commons Attribution - Pas d'Utilisation Commerciale - Pas de Modification 4.0 International. 


\title{
Des références pour des pratiques de formation Étude d'une situation de formation technologique supérieure initiale
}

\author{
Becerril Ortega, R., \\ Université Toulouse III, Laboratoire DIDIST (DIdactique de DIsciplines Scientifiques et Technologiques), Equipe DESEI \\ (Didactique et Enseignement de Sciences Expérimentales et de l'Ingénieur), CREFI-T, raquel.becerril@gmail.com
}

\section{Calmettes, B.}

IUFM Midi Pyrénées, DIDIST, DESEI, CREFI-T et GRIDIFE IUFM Midi-Pyrénées, Université Toulouse II, Le Mirail.

\author{
Fraysse, B., \\ INSA Toulouse, DIDIST, DESEI, CREFI-T
}

\section{Lagarrigue, $P$.,}

CRF Champollion, LGMT (Laboratoire de Génie Mécanique de Toulouse)

\begin{abstract}
References for training practices. A study of the situation of initial higher technological training.

This research focuses on the relations between professional context and higher technological education context during the development of a machine tool simulator. The conceptual framework outlines the context of higher technological education and articulates the didactical concepts, such as the transposition and the references in the composition of educational situations. In this perspective, an analysis of the epistemological roots of practical knowledge is presented, through the specific disciplinary action and the relationship of such knowledge with professional practice. The methodology is driven by the choice of learning content, through two interviews with two teachers and two simulator designers and then by analysing the development of teaching content (the "knowledge to teach") in two cases. The adopted methodology involves a number of tools developed within professional education: professional contexts analysis and the situation's conceptual structure. The results presented provide an understanding of the transpositional process through computer simulation and introduce some possible ways of operating that give continuity to this work.
\end{abstract}

KEYWORD

Professional didactics, higher education, references, transpositional process, practical knowledge, computer simulation.

\section{Introduction}

Lorsqu'on s'intéresse à la formation des ingénieurs et des techniciens supérieurs, on constate les rapports étroits que ces filières entretiennent avec le monde industriel, permettant de qualifier ces formations de professionnalisantes. Dans ce contexte, l'apprentissage porte sur une activité, future et anticipée, et non pas sur des savoirs. Pourtant l'organisation de cette activité anticipée s'appuie sur des savoirs (au sens le plus général de ressources à disposition des acteurs), qui sont de deux sortes, des savoirs académiques et des savoirs tirés de la pratique professionnelle. De plus, l'apprentissage 
est lui-même une activité, surtout quand il comporte beaucoup de Travaux Pratiques (TP) et de travaux sur simulateur. Comment s'articulent ces deux types de ressources dans l'apprentissage de l'activité?

Le statut des savoirs pratiques dans les cursus de formations à l'université est lié à la question de la professionnalisation mais aussi au modèle universitaire et à la discipline concernée.

- La modélisation de l'université, proposée par Lessard et Bourdoncle (2002), indique qu'il n'existe par a priori de « freins » de l'institution' à la professionnalisation.

- La discipline concernée par cette étude est la technologie, et d'une manière plus spécifique l'enseignement en génie mécanique (sciences de la production industrielle) aux Machines Outils à Commande Numérique (MOCN).

Les situations de formation étudiées se présentent en partie sur un simulateur informatique. Le cadre conceptuel convoque la théorie de la conceptualisation dans l'action en relation avec le statut des savoirs pratiques dans une perspective de développement de compétences. La spécificité de la technologie permet d'avancer ensuite l'existence de rapports entre le contexte professionnel et le contexte de la formation. Ces rapports sont analysés à partir de la demande d'habilitation de la formation visée. Le cadre conceptuel présente aussi le rôle de la simulation dans les formations professionnelles (ou professionnalisantes) et permet d'introduire la problématique concernant l'étude des processus de transposition dans ces formations technologiques supérieures.

Concernant cette transposition pour la formation avec simulateur, deux références sont explorées: les situations professionnelles et les savoirs issus de la recherche scientifique.

La méthodologie présente les conditions d'étude de la relation entre les contextes et analyse des références pour la transposition.

Les résultats et leurs discussions constituent finalement une contribution à l'étude des références pour les pratiques de formation.

\section{1.- Cadre conceptuel de l'étude}

\section{Le rapport entre savoirs théoriques et pratiques dans la formation initiale profession- nalisante}

Une question vive, centrale dans ce travail, consiste à analyser les relations entre les savoirs théoriques et les savoirs pratiques dans une formation qui se veut professionnalisante ${ }^{2}$. Hanh (2007) reconnaît, dans une perspective de formation en alternance, les difficultés que rencontrent des professeurs pour intégrer des connaissances théoriques dans le développement professionnel. Pour autant, Decomps et Malgaive (1996) signalent l'importance des savoirs pratiques, éléments centraux pour garantir la professionnalisation: "Le savoir pratique devient le principe même de l'alchimie des compétences, transformant des connaissances qui resteraient « livresques » à l'issue d'une formation purement scolaire en connaissances « opératoires parce que devenus outils pour l'action» (Ibid., p. 62).

La théorie de la conceptualisation dans l'action développée en didactique professionnelle permet de prendre en compte l'existence, du point de vue de l'apprentissage d'une personne, d'une dialectique entre ces deux types de savoirs. Pastré (2005) présente la connaissance sous deux formes imbriquées dans la même structure cognitive: une forme prédicative et une forme opératoire ${ }^{3}$. Il indique qu'une

1. La notion d'institution est prise ici sous l'acception développée par Chevallard (2003, p 83). L'institution est « un dispositif sociale «total » [...] qui permet et impose à ses sujets, [. . .] la mise en jeu de manières de faire et de penser propres ». Dans ce cas, il s'agit d'une institution de formation universitaire.

2. Le terme professionnalisante est utilisé par Cartonnet (2006) pour se référer au statut particulier des formations technologiques supérieures initiales avec une forte visée professionnelle.

3. Selon Pastré $(2005, \mathrm{p} 8)$ « [...] il existe deux formes de la connaissance, l'une de nature prédicative, qui s'exprime 
même connaissance peut, « soit s'investir dans l'action pour l'orienter, soit se constituer en un savoir socialement établi, qui pourra être transmis comme un patrimoine ».

Du côté d'une personne agissant, en suivant la théorie de la conceptualisation dans l'action, se trouvent imbriqués un modèle opératif, « représentation que se fait un sujet d'une situation dans laquelle il est engagé pour la transformer » (Pastré, Mayen, \& Vergnaud, 2006, p. 161), et un modèle cognitif, « en tant que représentation qu'un sujet se fait d'un domaine en termes d'objets, de propriétés et de relations, indépendamment de toute action de transformation portant sur ce domaine » (Ibid., p. 161). Selon Pastré (2005), dans l'apprentissage, on peut trouver deux grandes modalités d'articulation entre ces modèles: soit ils sont appris de manière indépendante, « avant que l'acteur, confronté à la pratique de l'activité, n'élabore son modèle opératif », soit ils sont appris simultanément, quand l'acteur suit un apprentissage sur « le tas».

Le rapport entre les savoirs théoriques et pratiques est présenté ici en relation avec la professionnalisation des formations universitaires et l'enjeu des enseignements pour l'élaboration chez l'apprenant des différents modèles.

\section{La spécificité de la formation universitaire technologique}

Les études didactiques dans l'enseignement supérieur technologique ne sont pas très nombreuses. Cartonnet (2000) étudie la formation des ingénieurs comme concepteurs de produits industriels, en s'intéressant notamment à l'organisation des données technologiques pour la conception et à son utilisation dans la formation. Ainsi, il présente un exemple que nous appliquons au contexte de l'apprentissage de la mise en œuvre d'une machine-outil à commande numérique. Cartonnet indique que « le mode d'enseignement par les travaux pratiques nécessite en effet un double regard didactique, disciplinaire et professionnel ». En TP de technologie, l'étudiant doit comprendre le fonctionnement d'une machine-outil à commande numérique (les différentes parties, les modes de fonctionnement, etc.) et il doit aussi apprendre à l'utiliser comme un opérateur sur machine. Et pour cela, il doit maîtriser des situations de réglage, de positionnement de pièces, de mesure d'outils, etc. Cartonnet rapproche de fait les entrées : par les didactiques disciplinaires et par les didactiques professionnelles. Ces dernières donnent importance à l'analyse de l'activité en vue de la formation de compétences professionnelles.

Le simulateur a été développé pour des raisons de coût mais aussi pour des raisons de formation. L'enseignement est très dirigé pendant les séances de formation sur MOCN pour éviter d'éventuels accidents. Les formateurs ont donc envisagé le développement d'un simulateur de machine permettant de donner davantage d'autonomie aux étudiants, de transformer une formation qui pourrait être trop théorique en une formation plus pratique. Les formateurs envisagent ainsi une pédagogique plus constructiviste, grâce aux possibilités du simulateur.

A priori, l'apprentissage par simulation est un apprentissage par l'action et par les situations de travail (Pastré, 2005). La simulation reproduit (partiellement) une situation professionnelle et permet un apprentissage transférable à la situation prise comme référence. Cependant, la reproduction est bien partielle puisque « la situation de simulation [...] a des caractéristiques propres qui achèvent de modifier le contexte de travail » (Leplat, 2006, p 38).

Samurçay (2005) a mis en lumière le double processus de médiation dans les formations avec simulateur entre l'apprenant et l'instructeur. Elle distingue deux moments dans la médiation prise en charge par l'instructeur: la transposition, en tant que rapport vers la situation de référence, et la gestion des situations. La perspective ouverte par Samurçay (2005), conduit à poser la question de la transposition dans les situations de formation utilisant des simulateurs informatiques.

par des énoncés susceptibles de s'organiser en savoirs, l'autre de nature opératoire qui voit dans la connaissance une adaptation intelligente aux situations [...]». 


\section{La prise en compte des processus transpositifs}

L'introduction des notions relatives aux débats autour des processus de transposition met en relation le statut des savoirs pratiques et la question des références dans le choix des situations de formation.

Le statut des savoirs pratiques a été pendant longtemps au cœur du débat sur le processus transpositif. Selon certains auteurs ce processus était exclusivement adapté aux savoirs issus de la théorisation et de l'axiomatisation des savoirs théoriques. Mais Joshua (1998) alerte sur les possibles dualismes provoqués par la division dans la terminologie de savoirs, entre théorie et pratique, qui contribuent à ennoblir la partie conceptuelle au détriment de la partie « active » et « expérientielle ».

La question de la référence peut être étudiée dans une perspective d'étude de processus transpositifs, en relation avec les approches épistémologiques des savoirs.

Pour Chevallard (1985), dans ses premiers travaux, les savoirs savants disciplinaires constituent la référence transpositive pour les savoirs à enseigner. Puis Chevallard (1994), dans le cadre de l'élargissement de la théorie de la transposition à d'autres réalités disciplinaires, pose le problème de l'articulation des pratiques et des savoirs, de l'écologie des savoirs et du statut du savoir. L'auteur propose de s'intéresser, dans le cadre de la transposition à la « trinité »: domaine de réalité, pratique, savoir. Deux difficultés apparaissent:

- La première est liée au choix du domaine de réalité et des pratiques (vivantes) qui vont être transposées dans une institution, (légitimité).

- La deuxième difficulté porte sur la construction dans la classe d'un domaine de réalité dans lequel opèrent les pratiques, (pertinence).

L'entrée proposée par Martinand (1981) par les pratiques sociales de référence est reprise dans diverses publications. Martinand (2003) vise à « expliciter la signification de choix dans la conception, l'essai et l'évaluation de projets d'enseignement ». Selon cet auteur, « la problématique de la référence n'est pas forcément opposée à celle de la transposition didactique ». Mais il convient d'opérer la distinction entre les deux problématiques. D’abord elles sont nées et développées au sein des disciplines différentes (les sciences expérimentales et la technologie d'une part et les mathématiques d'autre part). Ensuite, leur échelle d'application est différente: la notion de pratique sociale de référence permet de passer d'une « transposition restreinte (entre savoir savant et savoir enseigné) à une transposition générale (entre pratiques de référence et activités scolaires) ». Mercier (2002) critique ce positionnement qui consiste, selon lui, à oublier l'enjeu principal des phénomènes transpositifs, qui ne sont pas le produit de la référence mais celui du système d'enseignement.

Ces différentes approches sont étudiées en didactique comparée. Schubauer-Leoni et Leutenegger (2005) proposent une alternative permettant d'articuler les perspectives. Selon ces auteures, la didactique professionnelle étudie la transposition didactique dans un processus de création de contenus d'enseignement «à partir d'un ensemble de savoirs en acte manifestés dans la pratique », savoirs qui doivent être identifiés dans des pratiques efficaces. Dans leurs travaux, Rogalski et Samurçay (1994) relèvent deux voies possibles de transposition (de pratiques) : à travers les savoirs savants constitués par la théorisation et l'axiomatisation, et à travers des savoirs de référence de pratiques professionnelles. Rogalski et Samurçay répondent aux questions de Chevallard sur la pertinence et la validité épistémologique du choix de contenus: «Le savoir de référence doit être pertinent par rapport à la tâche concernée et pas seulement légitime par rapport à l'institution : [...] dans le cas de la formation professionnelle [...] il faut distinguer légitimité et pertinence. [... La] formation doit être pertinente par rapport aux objectifs fixés et aux tâches attendues. La légitimité se tient du côté de l'institution, la pertinence du côté de l'épistémologie et de l'action » (Ibid, p. 36).

Pastré (1999) propose une perspective extensive de la transposition didactique: « toute situation de référence, scientifique ou professionnelle appelle à des transformations lorsqu'on l'utilise comme situation d'enseignement et d'apprentissage: simplification, suppression de certaines variables, choix 
de situations prototypiques, etc. ». Cette approche permet ainsi de concilier le rapport à une référence dans l'élaboration des contenus de formation, incontournable dans le processus transpositif et le rapport institutionnel, propre à toutes les situations d'enseignement/apprentissage, voire didactiques.

L'étude de la transposition que nous effectuons s'intéresse à l'évolution d'un simulateur de machine-outil à commande numérique en fonction des besoins des apprenants. Pour cela, nous considérons à la suite de Samurçay (2005) une transposition et une conception de situations de formation avec comme double objectif de « conserver les fonctionnalités des situations de référence (Rogalski, $1995,1997)$ et de gérer la complexité par un processus de décomposition des connaissances et des pratiques, et de recomposition de compétences ».

L'enjeu de notre approche par rapport aux travaux précédents est donc:

- D'une part, tout en s'appuyant sur les bases de la didactique professionnelle, de prendre en compte le contexte de la formation initiale. En didactique professionnelle, il s'agit plutôt de formation continue. Le contexte dans lequel nous nous situons ici est de la formation initiale technologique supérieure à vocation professionnelle.

- D'autre part, il s'agit d'étudier le processus transpositif des situations professionnelles et les voies de modélisation des situations didactiques avec simulateur.

Notre cadre conceptuel repose sur l'existence des savoirs provenant de deux références: les savoirs constitués par la théorisation et l'axiomatisation, et les savoirs de pratiques professionnelles. Nous indiquons aussi l'importance dans une formation avec simulateur, d'analyser les voies de modélisation possible de ces savoirs afin de permettre le développement de compétences chez les acteurs.

Nous rappelons enfin le contexte de la formation: initiale, technologique et supérieure. Ce contexte conduit à des limites pour l'acquisition de compétences professionnelles.

\section{Les objectifs visés par la formation : acquérir des savoirs pour la pratique profession- nelle avec un simulateur de machine}

L'étude des processus transpositifs dans le contexte de la formation technologique supérieure est naturellement liée aux objectifs visés par l'institution concernée et aux caractéristiques disciplinaires de la formation.

En ce qui concerne les objectifs de la formation, nous allons présenter dans un premier temps, une analyse de documents officiels de la filière concernée. Par rapport à la discipline, nous poursuivrons en présentant quelques dimensions des courants épistémologiques des savoirs des ingénieurs.

Dans le cas des formations technologiques supérieures, cadre de notre recherche, l'analyse de la demande d'habilitation (1999) de la formation intitulée «Licence des sciences de la production industrielle » montre un lien fort entre le contexte professionnel et celui de la formation.

Nous y trouvons d'abord un axe prioritaire consistant à « proposer dans le domaine de la production industrielle une formation appliquée utilisant les techniques et les concepts actuels, cette formation n'ayant pas d'équivalent à ce jour dans notre région ». Pour ce faire, les étudiants sont « titulaires d'un DUT ou d'un BTS ${ }^{4}$ ». C'est-à-dire, étudiants ayant une expérience de formation en alternance (stage ouvrier) et qui ont donc développé a priori un début de modèle opératif (pour les situations visées en formation).

Nous trouvons ensuite, dans ce même document officiel, deux références à la formation avec machine-outil, conventionnelle ou numérique.

La première est indiquée dans le syllabus de l'unité d'enseignement «fabrication » (module 5). Il s'agit de «familiariser les étudiants aux possibilités des machines outils conventionnelles: cinématique des machines, surfaces obtenues (forme, précision, état de surface) ». Même si l'étude visée dans

4. Diplôme Universitaire de Technologie (DUT), Brevet de Technicien Supérieur (BTS) 
la présente recherche est la formation aux machines outils à commande numérique, l'introduction dans la formation d'un module dont le contenu porte sur les machines outils conventionnelles ${ }^{5}$ a des conséquences sur l'acquisition de certaines compétences, notamment celles concernant les effets des mouvements effectués par la machine-outil conventionnelle sur la pièce.

La deuxième est relevée au niveau du descriptif du module 6 « Sciences de la production », dans deux unités d'enseignement:

- «FAO (fabrication assistée par ordinateur): il s'agit de voir la programmation des machines outils à commande numérique par l'intermédiaire de logiciels de fabrication »,

- «Commande Numérique: l'objectif est de présenter les possibilités d'un centre d'usinage et d'un tour à commande numérique et d'amener les étudiants à un niveau de connaissance leur permettant d'être autonomes devant ces moyens de production ».

Dans ce cas, la répartition des volumes horaires est significative. Alors qu'au total on compte 12 heures de cours (6 pour l'unité «Fabrication » et 6 pour l'unité « Commande Numérique ») et 12 heures de Travaux Dirigés (pour l'unité « Fabrication »), on compte 48 heures de Travaux Pratiques: 18 pour l'unité « Fabrication » qui se déroulent sur machine conventionnelle, 18 pour l'unité «FAO » qui se déroulent face à un ordinateur, et 12 sur machine-outil à commande numérique. Nous faisons l'hypothèse qu'un volume horaire en Travaux Pratiques aussi important est lié au fait qu'il s'agit d'une formation à visée professionnelle.

Dans la perspective de la formation initiale, les étudiants sont censés acquérir plusieurs dimensions de compétences (Samurçay, 2005) : établir un diagnostic sur les différents modes de fonctionnement de la machine en fonction de qualités requises sur les pièces finies, acquérir des schèmes de contrôle manuel et automatique de la machine, apprendre à démarrer, régler, arrêter, calculer les distances entre la broche et la pièce, interpréter et gérer les alarmes telles que les arrêts de puissance, etc.

L'institution universitaire est donc porteuse d'orienter la formation vers des pratiques professionnelles, les objectifs de la formation sont exprimés en termes de compétences.

\section{Étude des références pour des pratiques de formation}

Notre étude a pour objet de clarifier la question de la référence dans le processus de transposition opéré dans le contexte de la formation technologique supérieure avec un simulateur.

La méthodologie et la discussion des résultats de présent travail s'organisent autour de ces deux questions:

- Quelles relations entre le contexte professionnel et le contexte de la formation dans le cas de la formation technologique supérieure?

- Quelles références dans la construction des savoirs pour cette formation?

\section{2.- Méthodologie de recherche}

La méthodologie est relative à deux étapes dans la recherche. La première conduit à éclairer la dialectique entre le contexte professionnel et le contexte de la formation universitaire. La deuxième permet d'identifier les sources des savoirs qui sont à l'origine de la transposition.

Les données recueillies sont qualitatives, issues des entretiens construits à partir du cadre conceptuel, des enregistrements vidéo des situations professionnelles ou des documents écrits

5. Une machine-outil est capable de maintenir un outil et lui imprimer un mouvement afin de tailler, découper ou déformer un matériau. Les machines outils conventionnelles sont celles contrôlées manuellement. Les machines outils à commande numérique (MOCN) disposent d'une commande numérique, c'est-à-dire une armoire de commande recevant le programme d'usinage sous forme d'un ruban perforé, d'une bande magnétique, ou de données issues d'un ordinateur. 
(référentiels de compétences, ouvrages universitaires, etc.). Les analyses menées sont aussi qualitatives, s'appuyant sur une « démarche discursive et signifiante de reformulation, d'explicitation ou de théorisation d'un témoignage, d'une expérience ou d'un phénomène ».

\section{1.- À propos de la dialectique entre contexte professionnel et contexte de la formation universitaire}

L'analyse de documents institutionnels (la demande d'habilitation) avait permis de caractériser le rapport fort entre contexte professionnel et formation universitaire.

Deux entretiens avec des enseignants (notés P1 et P2 par la suite) de cette filière permettent de préciser ce lien. Les entretiens visent la compréhension des choix de contenus et de leur mise en œuvre en classe, en relation avec le futur contexte professionnel des acteurs de la formation. Ces professeurs ont été choisis selon deux critères: l'expérience en formation et le « poids » dans l'institution.

- Un professeur expérimenté (20 ans d'expérience en enseignement), avec beaucoup de «poids institutionnel » et des responsabilités dans l'élaboration de curriculums (P1),

- Un maître de conférences, que l'on peut qualifier de novice au niveau de l'ancienneté dans l'enseignement dans la filière considérée (5 ans), sans responsabilités au niveau institutionnel (P2).

Le passage des entretiens a été différé. D'abord, l'entretien avec P1 a permis de relever certains aspects concernant son fort rôle institutionnel. Afin de compléter les résultats, nous avons choisi un deuxième enseignant, P2, dont les caractéristiques (novice et avec un rôle institutionnel moins influent) permettent d'éclairer les questions de recherche.

Ces entretiens sont conçus comme complémentaires puisque les deux professeurs travaillent dans la même filière de formation et assurent des cours sur machines outils à commande numérique (MOCN) et en fabrication assistée par ordinateur (FAO).

Deux dimensions de la tâche proposée aux étudiants ont été abordées:

1 - La première est relative au choix des contenus : quels sont les objectifs de la séance de formation? Quels sont les problèmes posés (et l'origine de ces problèmes)? Quelles sont les modalités de mise en œuvre ? Comment et à quel moment est envisagée l'évaluation? Il est important de signaler que pendant l'entretien, les questions relatives à cette première dimension ont été orientées vers la recherche des liens entre les deux contextes, professionnel et de la formation.

2-La deuxième s'intéresse aux supports matériels utilisés pendant la formation et à l'origine de ces supports: le simulateur, le cahier de TD, les polycopiés, etc. Il s'agit de comprendre le rôle des enseignants dans l'élaboration des cours dans l'institution concernée.

La méthode d'analyse des entretiens s'inspire de l'analyse catégorielle de contenus. Au final, cinq catégories ont été identifiées, concernant le rapport entre les contextes professionnel et de la formation. Les quatre premières sont liées aux aspects essentiels de tout projet didactique et éducatif:

- La mise en œuvre des contenus en classe,

- Les consignes données par l'enseignant pendant la situation de classe,

- Les objectifs de formation envisagés par les enseignants,

- La pratique professionnelle future des étudiants.

Une dernière catégorie apparaît, à propos de la relation entre l'entreprise et le contexte de formation.

\section{2.- À propos des sources des savoirs pratiques et théoriques}

Suivant les conclusions du cadre conceptuel, les sources de références sont les savoirs issus des 
contextes de travail et les savoirs élaborés par la théorisation et l'axiomatisation (Rogalski, \& Samurçay, 1994).

Pour accéder aux savoirs issus de contexte de travail, il s'agit d'élaborer la structure conceptuelle de la situation d'usinage de pièces avec des Machines Outils à Commande Numérique (MOCN). Pastré (1999), en didactique professionnelle, utilise le concept de structure conceptuelle d'une situation qu'il explique comme « un ensemble des concepts organisant l'action et servant à la guider ».

Pastré (2005) définit la structure conceptuelle d'une situation par les éléments que la composent:

- Des concepts organisateurs, permettant d'établir un diagnostic de la situation,

- Des indicateurs, donnant une valeur actualisée aux concepts,

- Des classes de situations que l'on peut analyser à partir de la valeur donnée aux concepts organisateurs.

Ces classes de situations vont spécifier le répertoire des procédures (ou des règles d'action) à utiliser et des stratégies attendues, en fonction du niveau de conceptualisation auquel a accès un opérateur.

Ainsi, une analyse qualitative de l'activité du travail, au sens ergonomique, a été effectuée en s'appuyant sur différentes données: les référentiels professionnels, un entretien avec un chef d'atelier des machines outils à commande numérique et des entretiens (trois) et une observation « en activité » avec un opérateur expert.

L'analyse s'est déroulée en trois étapes:

1. L'analyse globale du travail

Il s'agit de comprendre les aspects liés à l'organisation sociale du travail, aux différents acteurs et à leurs rôles respectifs dans le processus de fabrication industrielle. L'entretien a été élaboré en prenant comme référence les tâches prescrites par les référentiels de la formation professionnelle. L'entretien a été mené avec un chef d'atelier, choisi pour sa vision globale du processus, en fonction de son expérience, et de son parcours de formation. En effet, il s'agit d'un acteur de la formation continue en entreprise, avec une longue expérience « au pied des machines».

2. L'analyse de l'activité d'un opérateur expert

L'opérateur expert travaille en autonomie. Il effectue lui-même la programmation des pièces à partir d'une gamme ${ }^{6}$. Avec quarante ans d'expérience, il a vécu le passage des machines outils conventionnelles à l'automatisation par les MOCN. Un premier entretien et un film de l'opérateur en situation d'usinage, avec des explicitations verbales qui accompagnent son geste, ont été réalisés. L'analyse des données permet de situer l'activité de l'opérateur dans l'ensemble de processus de fabrication industrielle et de construire une première structure conceptuelle de la situation d'usinage des pièces avec des MOCN.

\section{Vérification de la structure conceptuelle élaborée}

Un entretien approfondi avec le même opérateur a été mené, après l'analyse précédente. Cela a permis l'identification des classes de situations et les indicateurs permettant à l'opérateur d'agir dans diverses circonstances.

Le processus transpositif dans le cadre du développement d'un simulateur pour la formation en contexte universitaire de formation technologique supérieure est présenté dans deux cas particuliers : l'enseignement des effets de la dilatation de la broche (élément mobile de la machine) d'une machine-outil et l'enseignement des repères de la machine dans sa mise en fonction par l'opération de réglage. Pour compléter cette perspective, un entretien avec les concepteurs du simulateur a aussi été mené.

Le développement du simulateur est envisagé comme le résultat d'une transposition didactique matérielle (Calmettes, 1997), ce qui permet de voir le simulateur dans l'optique des « savoirs à ensei-

6. Une gamme est un plan de réalisation de la pièce 
gner».

Pour accéder aux savoirs élaborés par les scientifiques, les ouvrages universitaires, la thèse de P2, ainsi que les entretiens énoncés précédemment, avec deux professeurs (P1 et P2) sont étudiés. Les ouvrages universitaires permettent d'accéder aux productions scientifiques et à leurs relations avec la modélisation effectuée par le simulateur. Le savoir étudié est relatif à l'évolution temporelle de la broche. La présentation de ce savoir grâce au simulateur est mise en relation avec les résultats de recherche dans le champ de génie mécanique.

\section{3.- Résultats}

D’une manière générale, l'analyse des situations de formation par la simulation informatique s'appuie sur les différentes « sources » des savoirs enjeu et sur l'étude du rapport entre le contexte de travail et celui de la formation dans le cas d'une filière universitaire technologique.

Plus spécifiquement, deux situations didactiques sont étudiées:

- La première, en relation directe avec le contexte professionnel utilise une méthodologie originale inspirée de la didactique professionnelle. Il s'agit d'analyser les processus de transposition à partir de la structure conceptuelle de la situation visée.

- La deuxième est en relation avec des savoirs issus du contexte de la recherche universitaire technologique.

\section{1.- Le rapport entre le contexte de travail et de formation dans l'élaboration de contenus}

Il semble que le rapport avec l'institution dans le choix des contenus de formation, laisse aux enseignants une certaine liberté. La demande d'habilitation est censée servir de référence pour la formation. Cependant P1 qualifie ce document de «souvent très court » et précise que selon lui sa fonction est plutôt à mettre en relation avec les coûts générés par étudiant.

Le rapport entre le choix de contenus de formation et le contexte professionnel futur des étudiants est analysé sous différents axes:

\section{Les relations entre les acteurs de la formation et les acteurs des entreprises}

P1 précise, par les relations entre entreprise et université, l'incidence du contexte professionnel sur le choix des contenus de formation: «quand on envoie les étudiants en stage, on voit ce qu'on leur demande, et en discutant avec les industriels on voit un petit peu de quoi ils ont besoin [...]. En règle générale, à chaque fois qu'on termine une soutenance de stage, on demande aux industriels ce qu'ils souhaitent que nous améliorions dans la formation des étudiants [...]».

P2 ne s'exprime pas sur ce sujet. Il s'exprime plutôt en faisant référence à son travail de recherche, ce qui soutient l'hypothèse selon laquelle P2, enseignant novice « préfère » parler de son expertise.

Les formateurs opèrent une mise en œuvre de problèmes issus du contexte professionnel et des problématiques de recherche. La transposition de ces problèmes est liée au milieu de la formation. Le décalage entre le type de problèmes choisis et la pratique professionnelle future est justifié par les enseignants, par une formation plus « large », voire « généraliste ». Les interactions entre le contexte professionnel et celui de la formation ont souvent lieu aux moments des évaluations de la formation, par exemple lors des présentations des stages que les étudiants ont effectués en entreprise.

\section{La pratique professionnelle future des étudiants}

On repère dans le discours de $\mathrm{P} 1$ certains éléments sur les possibles pratiques professionnelles des étudiants. On a relevé précisément que les étudiants ne vont pas exercer comme des programmateurs 
ou des régleurs dans leurs pratiques professionnelles futures. Il considère cependant indispensable une formation avec « une vue plus large ». P1 donne d'importance à tout moment au futur contexte professionnel.

P2 exprime moins spontanément des références au contexte de travail. Cependant lorsque la question de la pratique professionnelle des étudiants après leur formation est posée P2 répond: «ça je pense qu'ils vont pas directement usiner, par contre ils vont certainement travailler en amont, sur la préparation d'usinage, $[\ldots]$ donc parce que c'est important pour eux, [...] et ils peuvent être amenés à travailler également sur des pièces où on se rend compte qu'il $\mathrm{y}$ a des problèmes, donc qu'est-ce qu'il faut faire derrière pour résoudre ce problème, [...] ils ont souvent des projets à gérer, et ils ont besoin d'avoir ce recul pour pouvoir gérer ces projets ». Ce discours est en accord avec celui de P1, à la fois sur l'objectif général et sur les décalages nécessaires entre la formation et la pratique professionnelle.

\section{Les consignes données par les enseignants pendant la situation de classe}

Dans ces consignes, P1 explicite qu'il demande à ses étudiants de se positionner comme des professionnels - programmateur ou régleur - en fonction de la tâche requise: «Tout ça je l'explique aux étudiants, je leur dis: bon, maintenant j'ai ma pièce. Quand j'avais la casquette de programmateur, j'ai donné une origine programme à un endroit. Maintenant que j'ai une casquette régleur de machine, il faut que j'arrive à expliquer à la machine où se trouve l'origine programme par rapport à l'origine de la machine (origine mesure) $[\ldots] »$.

P2 explique le rôle de l'opérateur: «Moi, j'ai essayé d'être progressivement global, [...] j'essaie de leur dire que tout pouvait intervenir, y compris même l'opérateur de la machine, qui, lui, pouvait avoir une intervention, une influence sur le processus et sur la qualité de la pièce finale ». Les entretiens mettent en évidence l'importance pour la formation de ce « qui est faisable », les contraintes liées au milieu de la formation: temps, organisation de l'espace, conditions de sécurité. Cependant, le choix de contenus et les consignes mettent en relation directe les contenus proposés avec la pratique professionnelle.

\section{Les objectifs de formation envisagés par les enseignants}

Pour insister en ce sens et en relation avec le lien entre formation et contexte de travail, P1 indique: « [...] il est indispensable que la personne qui sort de chez nous en fabrication ait une vue relativement large, [... pour cela], on est quand même obligé d'avoir mis un peu les mains, dans le métier pour se rendre compte des difficultés. [...] c'est pas la peine avec les métiers qu'on demande maintenant qu'ils soient très performants pour sortir un programme pièce parce que c'est assez rare qu'on les appelle pour faire un programme pièce, ils vont appeler quand il y a un problème. [...] Et c'est là qu'il faut que l'étudiant sorte avec suffisamment de choses pour pouvoir se sortir de ce problème ». La formation initiale cherche à former à des compétences diverses non centrées sur une pratique professionnelle précise.

Les propos de $\mathrm{P} 2$ vont dans le même sens. Il précise à propos d'activités qu'il propose : «Certainement ils vont pas les faire eux directement, par contre ils savent que quand on doit intervenir sur tel ou tel paramètre, pour résoudre tel ou tel problème, $[\ldots]$ je leur fais faire les choix, pour leur montrer les différences. [...] L'idée c'est de leur montrer qu'on peut agir sur le processus et comment on fait, après ils ne le feront certainement pas, pas directement, par contre si dans un projet, il y a des problèmes au niveau d'usinage, pour tel problème ils sauront comment travailler ».

\section{La mise en ouvre des contenus de formation}

Les réponses de P1 orientent vers une approche pédagogique définissant une démarche qui va du concret au général et qui essaie d'extraire les fonctionnalités d'une machine afin de pouvoir travailler avec d'autres types de machines: «J'ai trouvé que ça se passait mieux avec les étudiants parce qu'on 
posait le problème, comment je peux faire ça et à partir du moment où je vois qu'est ce que je peux avoir comme solution, cette solution-là, on l'extrapole à d'autres types de machines ».

P2 discute plutôt de la motivation à l'apprentissage et de son sujet de thèse. Ces réflexions font souvent référence à son travail de recherche, et nous pouvons formuler l'hypothèse qu'étant P2 un enseignant novice, oriente davantage la formation vers les concepts qu'il maîtrise, et donc, sur ce qu'il a travaillé en thèse.

Par rapport à la généralisation des procédés à partir d'un cas particulier sur une machine, méthodologie de formation souvent explicité par les enseignants, P1 et P2 partagent l'idée que les situations restent les mêmes et $\mathrm{P} 2$ considère important l'utilisation des machines différentes pour apprendre le réglage: « [...] si on fait le réglage sur une machine de type fraisage, dans la séance suivante il faut qu'ils le fassent sur une machine de type tournage. Parce que derrière le réglage il y a exactement la même idée, mais après le réglage se fait de façon un petit peu différente ».

Dans les résultats suivants, nous allons caractériser la transposition que subissent les situations issues des deux références: le contexte professionnel et celui de la recherche, afin de les adapter à la formation, notamment par l'utilisation d'un simulateur informatique.

\section{2.- L'étude des références pour la construction des savoirs : deux voies d'exploration possibles}

Dans l'étude des références dans la construction des savoirs, deux voies sont explorées à travers l'analyse de deux cas particuliers. La première référence est le contexte de travail (les savoirs de la production industrielle). L'analyse porte sur le lancement d'une activité avec la Machine-outil à Commande Numérique $(\mathrm{MOCN})$, notamment son réglage initial. La deuxième référence correspond à la recherche scientifique (les savoirs de la recherche). Nous analysons la construction d'un savoir lié à l'évolution temporelle de la broche lors de son fonctionnement.

\section{Le contexte de travail, savoirs de la production industrielle}

L'étude de situations professionnelles permet d'accéder aux savoirs pratiques. Nous étudions la transposition de la pratique qui consiste à mettre en fonctionnement et à régler une MOCN. Cette situation constitue un des objectifs principaux dans les premières séances de formation.

Nous utiliserons la structure conceptuelle d'une situation comme un analyseur d'une forme de connaissance pragmatique. Selon Pastré (2006), le registre pragmatique permet la réussite de l'action et une conceptualisation à travers la mise en relation des prises d'information sur la situation et des règles d'action disponibles.

Dans la structure conceptuelle de la situation que nous construisons à partir des observations réalisées dans l'entreprise, deux concepts organisateurs de l'activité sont définis. « Ce sont les dimensions, extraites du réel qui vont permettre de fonder le diagnostic de la situation » (Vidal-Gomel, \& Rogalski 2007): la qualité de la pièce usinée et le choix de positionnement initial de la pièce.

La qualité de la pièce usinée:

Dans une situation professionnelle, le but est d'usiner les pièces dans les niveaux de tolérances requises. Le chef de qualité détermine la validité ou le refus des pièces usinées. Les pièces doivent être usinées en utilisant de manière optimale le temps, pour de raisons évidentes de coûts.

Ce concept organisateur, correspond à l'objectif principal de l'activité. On peut noter qu'il intervient à différents niveaux de l'organisation sociale du travail. La qualité de la pièce est en effet déterminée depuis le bureau de conception, et décrite dans la gamme que l'opérateur doit effectuer. L'opérateur contrôle la pièce afin de vérifier qu'elle est dans les tolérances requises, mais un contrôle extérieur est aussi effectué: «Le but il est là, d'essayer avec le moins d'outillage possible de réaliser la pièce, le but est de la faire le plus vite possible et bonne parce qu'on a un contrôleur qui est tatillon. » 
De plus, la qualité requise de la pièce est présentée comme la difficulté la plus importante dans le travail d'un opérateur: "Le plus difficile est de réaliser la pièce avec la précision, quelle que soit la forme qu'elle ait. Ça demande une concentration et de savoir les programmes qui vont bien, pour faire la précision et faire de la précision répétitive, en série $[\ldots] »$.

Ce concept organisateur a une visée pragmatique importante. Il permet à l'opérateur de fonder un diagnostic de la situation et d'orienter ces actions.

Dans le cas de la conduite des MOCN, on identifie deux paramètres associés à ce concept organisateur, en tant que variables mesurables ou calculables (Caens-Marty, Spegcona, Délépine, \& Girerd, 2004): la lecture de la tolérance requise dans le plan de la pièce, avant l'usinage et la mesure de la pièce usinée, après l'usinage.

On identifie aussi deux indicateurs, «En tant qu'observables, naturels ou instrumentés, qui permettent d'identifier la valeur que prennent les concepts organisateurs. L'équivalent d'une relation signifiant-signifié relie les indicateurs aux concepts » (Vidal-Gomel, \& Rogalski, 2007, p. 53).

Le premier indicateur est l'état de la surface de la pièce usinée. Quand un opérateur a fini d'usiner une pièce, l'état final de la surface de la pièce est un indicateur de la qualité. Il ne doit pas avoir de bavures, ou des facettes. Cet indicateur permet de fonder un diagnostic de la situation et d'orienter l'action, par exemple, vers le changement de l'outil: « [...] parce que l'outil fait toujours de la bavure, l'outil quand on taille c'est comme un couteau, quand on taille bien ça va, quand ça ne taille plus, il faut forcer. L'outil c'est pareil donc il force la matière et il fait de la bavure, donc quand on commence à avoir beaucoup de bavure on change l'outil ».

Le deuxième indicateur est le bruit. Il intervient en relation avec un paramètre, constitué par l'ensemble des conditions de coupe (vitesse de rotation, avance, etc.) qui contribuent à la qualité de la pièce. L'activité de l'opérateur consiste à prendre les valeurs données par la programmation et à effectuer une régulation en fonction des indicateurs fournis par la situation: bruit et surface de la pièce usinée. À partir de ces indicateurs, l'opérateur régule les paramètres tels que la longueur d'outil, la vitesse de coupe et l'avance pour retrouver un équilibre. Il faut signaler, que comme dans le cas de la mesure de la qualité, ces paramètres sont d'abord calculés par l'opérateur à travers des calculs analytiques. Prenons l'exemple du calcul de la vitesse de rotation: « [...] suivant l'outil qu'on utilise, on calcule la vitesse de rotation, il y a une formule. Alors la formule c'est: le diamètre de l'outil, multiplié par 3,14 et divisé par la vitesse de coupe ».

Celle-ci constitue une première approximation qui est régulée ensuite à travers des indicateurs prélevés du réel par l'opérateur: «On bouge plus ou moins la rotation pour voir... Parce que suivant la fraise qu'on utilise [...] la fraise longue a tendance à vibrer et la vitesse de coupe il faut la réduire, alors la réduire jusqu'à ce qu'on adapte la vitesse de coupe à l'avance aussi parce que tout est lié. [...] ça fait qu'on essaie de trouver l'équilibre ».

Nous avons établi les relations entre le concept organisateur de la qualité requise de la pièce, les paramètres qui interviennent, et les indicateurs permettant à l'opérateur de fonder un diagnostic de la situation.

\section{Le choix de positionnement de la pièce:}

Le premier positionnement de la pièce est défini dans la phase de conception et de programmation de la pièce. Un bon positionnement permet de gagner du temps, de la qualité et est économique. Cependant, dans des situations d'usinage de pièces complexes, les compétences des opérateurs experts peuvent s'avérer essentielles. Ainsi, le chef d'atelier dit que l'opérateur: « doit [...] prévoir comment faire la pièce pour éventuellement intervenir s'il y a un problème. Donc, il y a des connaissances du produit fini que l'opérateur doit avoir. [...]. Et puis il doit connaître très bien la programmation $\mathrm{CN} »$.

Les compétences de l'opérateur sur le positionnement de la pièce permettent d'établir un dialogue entre la programmation et l'usinage afin d'améliorer la fabrication des pièces. Ce dialogue ne s'arrête 
pas là. Il s'est avéré indispensable pour les programmateurs, d'avoir réalisé des usinages des pièces, pour comprendre leurs difficultés et programmer en fonction de celles-ci. Le premier positionnement de la pièce est étroitement lié à cette compréhension des difficultés des opérateurs.

Ce concept organisateur n'intervient en général dans l'activité de l'opérateur que dans le cas d'un dysfonctionnement (mais il constitue un élément central des modèles opératifs des novices).

La contextualisation de ce concept organisateur permet d'entrevoir son importance. L'opérateur, avec lequel l'entretien a été réalisé, travaille dans la fabrication en série de pièces simples (boîtiers pour l'électronique). Cela lui demande de préparer, programmer et usiner les plans de pièces reçus du bureau des méthodes. Avant de commencer l'usinage, l'opérateur doit positionner la pièce de manière à pouvoir la terminer dans les meilleures conditions. Pour cela il envisage toutes les opérations jusqu'à la fin de la phase d'usinage: "Dans l'usinage la difficulté est de savoir comment réaliser la pièce, comment commencer [...] il faut bien étudier au départ, comment on va réaliser toutes les opérations qu'on va réaliser sur la pièce, et ça, on n'attaque pas la pièce sans savoir comment on va la réaliser, comment on va la finir [...]». La stratégie mobilisée doit permettre de toujours maintenir la pièce: à travers un talon, un morceau de matière, etc. Cette capacité dépend, selon l'opérateur, de l'expérience : «Alors ça c'est à force d'usiner, on sait à peu près par où il faut commencer, par où il faut finir [...]. Et ça c'est à force de faire, c'est la personne qui le programme qui le voit ça ».

Nous avons identifié deux paramètres permettant d'évaluer la stratégie: la visibilité de la pièce et le nombre de déplacements de la pièce. La visibilité de la pièce permet d'associer une surface et la broche pour l'usinage.

Le nombre de déplacements doit être minimum: «Et après on usine, [...] (et) une fois que le programme est fini, on enlève une pièce on remet une autre et on recommence, et quand la pièce est serrée ou quand elle est positionnée j'essaie de faire le maximum de cotations possibles sans démonter, pour aller plus vite».

Ce paramètre permet à l'opérateur d'expliciter la règle d'action qui consiste à prioriser un changement d'outil à un déplacement de la pièce, pour des gains économiques: « Ça fait que quand je fais une face $[\ldots]$ je fais le maximum d'opérations sans bouger la pièce. Quand on fait une pièce dans laquelle il y a beaucoup d'usinage, sans bouger la pièce il faut faire le maximum d'opérations sur une face. [...] Parce que le changement d'outil est vite fait, plus vite que de changer la pièce. Maintenant avec les machines, un changement d'outil ça veut dire quoi, deux secondes ! [...] C'est là qu'on gagne plus de temps ».

Une situation de dysfonctionnement identifiée consiste à effectuer un plan de pièce dont les positionnements successifs ne tiennent pas compte de la forme de la pièce. Cela provoque des déformations qui affectent la qualité de la pièce finie.

Le positionnement de la pièce peut dépendre aussi de la matière utilisée. Par exemple, dans le cas de l'aluminium, il faut prendre en compte le sens de fibres dans l'usinage de la pièce.

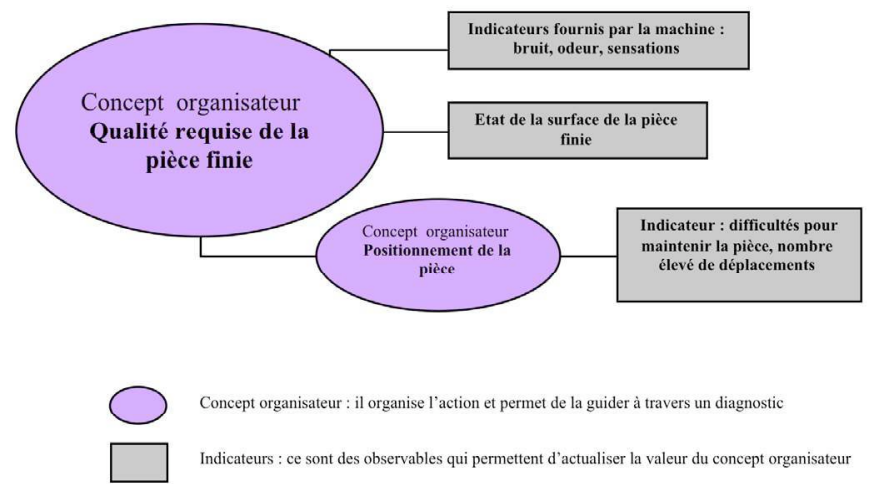

Figure 1: Structure Conceptuelle de la Situation d'usinage des pièces avec MOCN

Figure 1: Conceptual Structure of the Status of machining parts with MOCN 
La figure 1 représente la Structure Conceptuelle de la Situation d'usinage avec MOCN. La qualité de la pièce est le concept organisateur principal de l'activité des opérateurs sur MOCN, et le positionnement de la pièce est un organisateur secondaire, mobilisé dans le cas de dysfonctionnement pour les opérateurs experts et comme composante du modèle opératif chez les novices.

L'opérateur décrit cette procédure, concernant la mise en fonctionnement et le réglage d'une MOCN, comme étant indispensable à réaliser au début, afin de situer la pièce par rapport aux origines machines. Le réglage doit s'effectuer à chaque fois que (le modèle) la pièce change, car même si selon l'opérateur, les origines ne changent pas forcément: «le programme c'est plus le même, les outils des fois c'est plus les mêmes donc il faut faire les longueurs d'outils ».

Cette structure conceptuelle est validée lors de l'étude du réglage de la machine grâce à une vidéo de l'opérateur en action, accompagné des explications sur sa pratique.

La structure conceptuelle permet de mettre en relation des composantes de l'activité professionnelle avec des composantes des pratiques présentées en formation avec un simulateur. Dans ce sens, elle peut constituer un analyseur de la transposition didactique.

\section{La transposition dans la présentation du dispositif}

L'intérêt est porté au changement de représentation propre au passage d'une MOCN de type NUM vers un simulateur intégré dans un ordinateur, avec une interface différente, et aux changements de fonctionnalités produites par la modélisation de l'outil informatique. Dans l'analyse du simulateur nous avons repéré une transposition de type matérielle, dans la représentation que donne le simulateur des éléments de la machine.

En ce qui concerne la représentation de la fraise, le simulateur considère que l'équerre est parfaitement dégauchie, ce qui permet d'éviter une vérification lors de la prise de références dans la situation de réglage. Pendant l'opération de réglage, il faut, sur une machine, utiliser un outil palpeur pour aller repérer les points qui serviront d'origine. Si on avance trop avec le palpeur, on risque de le casser. Cependant, le simulateur ne représente pas la casse du palpeur et donc l'utilisateur peut le faire pénétrer dans la pièce et cela conduit à un défaut de puissance.

L'objectif de la présente recherche est d'étudier le processus transpositif de pratiques «efficaces » issues des contextes professionnels. La structure conceptuelle de la situation sert de référence afin d'« extraire » ce qui est commun à une classe de situations. Les analyses des entretiens effectués en contextes professionnel et universitaire convergent sur l'idée qu'un réglage de machines outils diffère d'une machine (du même type) à l'autre, change en fonction du type de machine, mais le principe reste le même. Ce principe commun correspond à la séquentialité des actions requises (mise en route, recherche des origines, calcul de vecteur, longueur d'outils) pour effectuer une opération de réglage. Dans l'analyse du processus de développement du simulateur, ses concepteurs déclarent avoir fait le choix de modéliser les fonctions que l'on trouve en général sur toutes les machines outils en respectant le langage de programmation de la machine de type NUM®.

L'analyse de l'activité d'un opérateur expert sur MOCN a permis d'établir la structure conceptuelle de la situation d'usinage des pièces. La mise en fonctionnement et le réglage de la MOCN ont été ensuite présentés comme des situations référées à cette structure conceptuelle par les concepts qui organisent l'activité : la qualité de la pièce et son positionnement initial. L'étude du simulateur a permis de caractériser la transposition opérée par les concepteurs afin de rendre cet instrument pertinent pour la formation. La transposition a été fondée sur les principes qui guident l'activité d'un opérateur expert: la séquentialité des opérations nécessaires, ainsi que les fonctionnalités génériques des MOCN. Nous relevons cependant certaines différences, d'ordre matériel, par exemple l'absence de possibilité de casse des outils. En conclusion, nous pouvons cependant affirmer que les transformations de la situation issue du contexte professionnel, relative à la mise en fonctionnement et réglage de la machine, conserve « les fonctionnalités des situations de référence (Rogalski, 1995,1997)». 


\section{La recherche scientifique, savoirs scientifiques}

Nous analysons ici les rapports entre le contexte professionnel et le contexte de la recherche, dans le cas d'un laboratoire en génie mécanique. Cette relation se traduit par des projets de recherche en collaboration, et dans le cas de ce laboratoire de production, par le souci principal de l'amélioration de la qualité des pièces usinées. Les laboratoires cherchent à élaborer des modèles de comportement des éléments des machines pendant la phase d'usinage des pièces. Les résultats des recherches servent donc à améliorer le cycle de production de pièces et profitent aux entreprises.

Nous allons analyser le processus de transposition d'un savoir théorique à un savoir enseigné par la modélisation effectuée grâce à un simulateur informatique.

Le savoir considéré est relatif à l'étude de l'évolution temporelle de la broche (élément mobile et changeable d'une machine-outil). Ce savoir est lié à la qualité de la pièce usinée et conduit à construire une modélisation du comportement de la broche.

La modélisation de ce phénomène à partir des travaux menés au sein du Laboratoire de Génie Mécanique de Toulouse (LGMT) permet aujourd'hui de calculer la variation de la longueur de la broche en fonction du temps et des conditions de coupe. La conséquence directe de cette variation dans la longueur de la broche sur la pièce usinée est la variation de la profondeur d'usinage.

Ce processus de transposition d'un savoir théorique en un savoir à enseigner correspond aussi à une spécificité dans la construction des savoirs propres à une équipe de ce laboratoire. Lors de l'entretien, P2 chercheur chargé de modéliser ce phénomène d'un point de vue de la recherche scientifique, explique la relation entre le phénomène et ses conséquences: «l'idée c'était une modélisation relativement pragmatique des défauts, [...] notre idée c'était de dire, que ce qu'on veut, c'est caractériser la flexion, c'est qu'on connaisse l'outil qu' on utilise et les conditions de coupe à utiliser pour la matière usinée, sur la machine où on usine, pourquoi passer par un intermédiaire qui prend de liaison, plutôt pris comme une inflexion, au lieu de prévoir directement les défauts d'usinage liés à la flexion de l'outil, donc les défauts d'usinage sur la pièce, en fonction des conditions de coupe. Donc, ça n'était pas la flexion de l'outil [...] mesurer les défauts auxquels était confrontée la pièce, en fonction des conditions de départ ». Dans son discours ce chercheur propose donc d'utiliser une modélisation qui atteigne directement les défauts d'usinage provoqués sur la pièce en fonction des conditions de coupe, au lieu de modéliser l'outil de coupe.

La figure ci-dessous représente les effets de ce phénomène sur le simulateur.

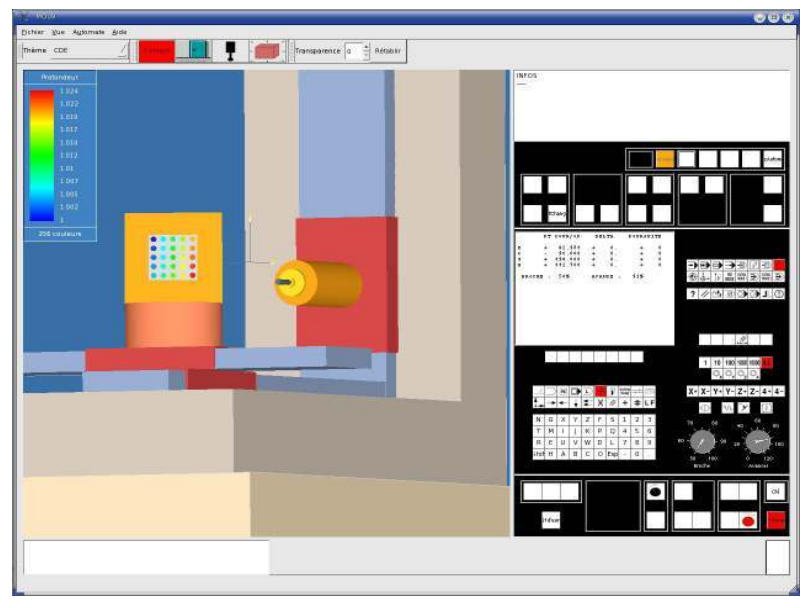

Figure 2: Défauts sur la pièce usinée montrés par échelle de couleurs. Copie d'écran du simulateur.

Figure 2: Defects on the machined part shown by color scale. Simulator Screenshot 
La modélisation effectuée avec le simulateur informatique permet d'afficher en échelle de couleurs, les différentes profondeurs dans la pièce, conséquences de la dilatation de la broche pendant la phase d'usinage. La représentation de ce phénomène est prise en compte avec une échelle de couleurs qui affiche les erreurs sur la profondeur d'usinage. Ces erreurs portent sur la différence entre une situation d'usinage dite « idéale », dans laquelle il n'y a pas de variation de longueur, et une situation considérée plus proche de la réalité. Par la transposition réalisée, le savoir à enseigner n'est plus le seul phénomène de dilatation de la broche, mais aussi les conséquences que ce phénomène produit sur la pièce usinée.

\section{La dilatation de la broche en contexte de travail}

L'opérateur expert explique également qu'en contexte de travail, ce phénomène de dilatation de la broche est identifié par ces conséquences au niveau de la pièce et la manière de compenser ce défaut. Dans une situation de travail, un opérateur expert identifie le phénomène de dilatation de la broche par des conséquences sur la pièce usinée (profondeur d'usinage notamment) et établit un processus de compensation pour que la pièce sorte dans les tolérances requises: «c'est surtout la profondeur quand ça bouge, comme ça, on contrôle la pièce si ça a bougé on rattrape avec l'origine ».

\section{La transposition dans la présentation du dispositif}

Dans le processus de transposition d'un savoir issu de la recherche scientifique, nous avons pris en compte le contexte du travail et celui de la formation. Nous relevons que le même concept organisateur, concernant la qualité de la pièce requise est mobilisé dans les deux activités. De la part de l'opérateur, c'est l'indicateur état de la surface de la pièce qui lui permet de déployer une stratégie de compensation afin d'atteindre la qualité requise. De la part des enseignants chercheurs, c'est une problématique, issue du contexte professionnel et en relation avec la qualité requise, qui est à l'origine du concept théorique. Dans ce sens, nous parlons d'un concept organisateur imposé par l'organisation du travail.

\section{Conclusion et perspectives}

Dans ce travail de recherche nous avons analysé les processus transpositifs qui ont lieu dans un milieu de formation technologique supérieur lors du développement d'un simulateur de machine-outil à commande numérique.

L'articulation des concepts didactiques, notamment la transposition et les références dans la constitution des situations de formation, a permis de positionner ce travail dans une perspective de formation initiale aux savoirs pratiques. Cet objectif d'acquisition des savoirs pratiques, nous l'avons vu, est appuyé depuis l'institution, par une déclaration générale d'objectifs et par la mise en place de formations orientées vers des pratiques. L'ancrage épistémologique de ces savoirs pratiques est double: la spécificité disciplinaire et le rapport avec la pratique professionnelle. Cet ancrage demande une étude des savoirs de référence par des modélisations issues de la recherche scientifique et du contexte de travail.

L'étude présentée s'intéresse d'abord au choix de contenus d'enseignement, grâce à des entretiens avec de professeurs et ensuite à l'élaboration de contenus d'enseignement (les " savoirs à enseigner ») dans deux cas particuliers. Ces cas ont été choisis pour leur représentativité: d'une part il s'agit de l'étude de la mise en fonctionnement et le réglage d'une MOCN qui a été décrite à partir de la pratique professionnelle, d'autre part c'est l'étude d'un phénomène de dilatation de broche qui a été modélisé en situation de recherche scientifique.

Dans un premier cas, nous avons utilisé la structure conceptuelle de la situation de mise en fonctionnement et de réglage de machine-outil à commande numérique, construite grâce aux observations de la pratique professionnelle, comme analyseur de processus transpositif. Nous avons centré notre 
attention sur la manière dont les concepts organisateurs (qualité de la pièce finie et positionnement de la pièce) sont modélisés par le simulateur.

Lors de l'analyse de la transposition du phénomène de dilatation de la broche par le simulateur, il s'agit de passer d'un phénomène produit sur une partie de la machine (la broche qui se dilate) à la conséquence qu'il produit au niveau de la pièce usinée. Cette transposition est liée à un processus épistémologique de construction des savoirs au sein de l'institution. La confrontation de cette modélisation avec les savoirs explicités par un professionnel met en lumière les différentes approches suivant les contextes: la pratique professionnelle dans l'usine, dans le laboratoire et dans le milieu de formation universitaire.

Dans ce travail de recherche, nous avons étudié le processus transpositif dans une filière de formation universitaire à vocation professionnelle. Nous avons pu établir des liens étroits entre la conception de la formation et la pratique professionnelle future et possible des étudiants. Deux voies de transposition ont été établies: celle de la théorisation et l'axiomatisation et celle à partir de la structure conceptuelle d'une situation. L'analyse du processus transpositif sera complétée par l'étude des situations didactiques de formation avec le simulateur informatique afin d'identifier, puis agir, sur les éventuels obstacles épistémologiques.

La dialectique entre le contexte professionnel et le contexte de la formation s'avère porteuse pour l'élaboration des concepts techniques (Vidal-Gomel, \& Rogalski, 2007). La prise en compte des problématiques industrielles par les laboratoires de recherche technologique, est une voie prometteuse dans l'étude de l'élaboration des concepts théoriques à partir des concepts pragmatiques (Pastré, 1999).

Une autre perspective ouverte par ce travail est la poursuite de l'analyse de processus transpositifs. Effectivement, l'analyse de la situation de formation avec simulateur en contexte universitaire technologique permettra de mesurer l'influence du contexte professionnel dans les situations effectivement mises en œuvres. Nous rejoignons la réflexion de Leplat (2006, p. 38) sur: « la situation de simulation n'est pas seulement une partie de la situation de référence, elle a des caractéristiques propres qui achèvent de modifier le contexte de travail ». Dans ce sens, nous avons avancé un premier pas dans l'analyse de ces caractéristiques propres des situations de simulation. Le contexte de la formation initiale par ces contraintes, institutionnelles, chronologiques, matérielles, constitue une voie très prometteuse. L'approche que nous proposons trouve son originalité dans la combinaison des réflexions et méthodes des didactiques professionnelles et des didactiques disciplinaires. Dans ce sens, elle constitue une heuristique prometteuse.

\section{RÉFÉRENCES}

Caens-Marty, S., Spegcona, A., Délépine, L., \& Girerd, S. (2004). Un simulateur pour répondre à des besoins de formation sur la taille de la vigne. Revue STICEF, 11 . http://sticef.univ-lemans.fr/num/vol2004/ delepine-02/sticef 2004 delepine 02.pdf [consulté le 27/5/2008].

Calmettes, B. (1997). Transposition didactique, une étude de cas en physique appliquée. Actes du sixième séminaire national de recherche en didactique de la physique, de la chimie et de la technologie, Lyon, Université Claude Bernard.

Cartonnet, Y. (2000). L'actualisation de la technologie structurale pour la formation de la technicité d'un concepteur de produits industriels. Mémoire d'HDR, Université Paris XI, Orsay.

Cartonnet, Y. (2006). Comment caractériser la valeur pre-professionnalisante des formations technologiques? In B. Fraysse (Ed.), Professionnalisation des élèves ingénieurs (pp. 87-103). Paris: L’Harmattan.

Chevallard, Y. (1985). La transposition didactique, du savoir savant au savoir enseigné. Grenoble: La pensée sauvage. 
Chevallard, Y. (1994). Les processus de transposition didactique et leur théorisation. In A. Gilbert, Y. Chevallard, J.L. Martinand, \& A. Tiberghien (Eds.), La transposition didactique à l'épreuve (pp. 135180). Grenoble: La pensée Sauvage Editions.

Chevallard, Y. (2003). Approche anthropologique du rapport au savoir et didactiques des mathématiques. In S. Maury, \& M. Caillot (Eds.), Rapport aux savoir et didactiques (pp. 81-104). Paris: Fabert.

Decomps, B., \& Makglaive, G. (1996). Les savoirs de l'ingénieur. In J.M. Barbier (Ed), Savoirs théoriques et savoirs d'action (pp. 91-100). Paris: PUF.

Hanh, C. (2007). Construire le lien entre pratiques professionnelles et savoirs théoriques dans l'enseignement supérieur. Education Permanente, n $172,39-44$.

Joshua, S. (1998). Des « savoirs » et de leur étude: vers un cadre de réflexion pour l'approche didactique. L'année de la recherche en sciences de l'éducation, Paris: PUF, 79-97.

Leplat, J. (2006). Les contextes en formation. Education Permanente, n 166, 29-48.

Lessard, C., \& Bourdoncle, R. (2002). Qu'est-ce qu'une formation professionnelle universitaire ? Conceptions de l'université et formation professionnelle. Note de Synthèse. Revue Française de Pédagogie, $\mathrm{n}^{\circ} 139,131-154$.

Martinand, J.-L. (1981). Pratiques sociales de référence et compétences techniques. A propos d'un projet d'initiation aux techniques de fabrication mécanique en classe de quatrième. In A. Diordan (Ed.), Diffusion et appropriation du savoir scientifique : enseignement et vulgarisation. Actes des Troisièmes Journées Internationales sur l'Education Scientifique (pp. 149-154). Paris: Université Paris 7.

Martinand, J.-L. (2003). La question de la référence en didactique du curriculum, Investigações em Ensino de Ciências, 8 (2). http://www.if.ufrgs.br/public/ensino/vol8/n2/v8 n2 a2.html, [consulté le 12/11/2007].

Mercier, A. (2002). La transposition des objets d'enseignement dans la définition de l'espace didactique, en mathématiques. Note de synthèse. Revue Française de Pédagogie, n 141, 135-171.

Pastré, P. (1999). L’ingénierie didactique professionnelle. In P. Carré, \& P. Caspar (Eds.), Traité des Sciences et Techniques de la Formation (pp. 465-480). Paris: Dunod.

Pastré, P. (2005). La conception des situations didactiques à la lumière de la théorie de la conceptualisation dans l'action. In P. Rabardel, \& P. Pastré (Eds.), Modèles du sujet pour la conception (pp. 73-108). Toulouse: Octarès Editions.

Pastré, P. (2006). Apprendre par l'action, apprendre par la simulation. Education Permanente, $\mathrm{n}^{\circ} 168$, 205-216.

Pastré, P., Mayen, P., \& Vergnaud, G. (2006). La didactique professionnelle. Note de synthèse. Revue Française de Pédagogie, $\mathrm{n}^{\circ}$ 154, INRP, 145-198.

Rogalski, J. (1997). Simulations : fonctionnalités ? validité ? In P. Béguin, \& A. Weill-Fassina (Eds.), La simulation en ergonomie : connaitre, agir et interagir (pp. 55-76). Toulouse: Octarès Editions.

Rogalski, J., \& Samurçay, R. (1994). Modélisation d'un « savoir de référence » et transposition didactique dans la formation de professionnels de haut niveau. In A. Gilbert, Y. Chevallard, J.L. Martinand, \& A. Tiberghien (Eds.), La transposition didactique à l'épreuve (pp. 35-71). Grenoble: La pensée Sauvage Editions.

Samurçay, R. (2003). Concevoir des situations simulées pour la formation professionnelle: une approche didactique. In P. Pastré, P., \& P. Rabardel, Apprendre par la simulation : de l'analyse de travail aux apprentissages professionnelles (pp. 53-71). Toulouse: Octarès Editions.

Samurçay, R. (2005). Concevoir des situations didactiques pour la formation professionnelle : une approche didactique. In P. Rabardel, \& P. Pastré (Eds.), Modèles du sujet pour la conception (pp. 53-72). Toulouse: Octarès Editions.

Schuabauer-Leoni, ML., \& Leutenegger, F. (2005). Une relecture des phénomènes transpositifs à la lumière de la didactique comparée. Revue Suisse de sciences de l'éducation année 27-3, Thema: didactique(s) entre continuité et réorientation, 407-427. 
Vidal Gomel, C., \& Rogalski, J. (2007). La conceptualisation et la place des concepts pragmatiques dans l'activité professionnelle et le développement de compétences. Activités, 4 (1), 49-84. http://www. activites.org.

\section{DOCUMENTS OFFICIELS}

Demande d'habilitation (1999) de la formation intitulé « Licence des sciences de la production industrielle » avec le $n^{\circ}: 384 \mathrm{~L} 2009$.

\section{RÉSUMÉ}

Ce travail de recherche s'intéresse aux rapports entre le contexte professionnel et le contexte de la formation technologique supérieure lors du développement d'un simulateur de machine-outil.

Le cadre conceptuel présente le contexte de la formation technologique supérieure et articule des concepts didactiques, tels que la transposition et les références dans la constitution des situations de formation. Dans cette perspective, une analyse de l'ancrage épistémologique des savoirs pratiques est présentée, à travers la spécificité disciplinaire et le rapport de ces savoirs à la pratique professionnelle. Concernant cette transposition pour la formation deux références dans la modélisation des savoirs sont explorées : les situations professionnelles et les savoirs issus de la recherche scientifique.

La méthodologie est portée parle choix de contenus d'enseignement, à travers deux entretiens avec deux professeurs et deux concepteurs du simulateur et ensuite par l'analyse de l'élaboration de contenus d'enseignement (les « savoirs à enseigner »). La méthodologie utilisée mobilise certains outils développés au sein de la didactique professionnelle: l'analyse du contexte professionnel et la structure conceptuelle de la situation. Les résultats exposés permettent de comprendre les modalités de transposition effectuées à travers la simulation informatique et annoncent des possibles voies d'exploitation qui donnent continuité à ce travail.

\section{MotS CLÉS}

Didactique professionnelle, formation universitaire supérieure, références, processus transpositifs, savoirs pratiques, simulateur informatique.

\section{RESUMEN}

Referencias pasa prácticas de formación. Estudio de una situación de formación tecnológica superior inicial. Este trabajo de investigación estudia las relaciones entre le contexto profesional y el contexto de la formación tecnológica superior que se dan en le marco del desarrollo de un simulador de maquina-herramienta.

El marco conceptual presenta el contexto de la formación tecnológica superior y articula conceptos didácticos como la transposición y las referencias en la constitución de situaciones de formación. En esta perspectiva, presentamos un análisis del anclaje epistemológico de los saberes prácticos, a través de la especificidad disciplinar y la relación entre esos saberes y la practica profesional. Acerca de esta transposición para la formación, se exploraron dos referencias de la modelización de los saberes: las situaciones profesionales y los saberes desde la investigación científica.

La metodología esta guiada por la elección de los contenidos de la enseñanza, por medio de dos entrevistas con dos profesores y con dos diseñadores de simuladores, y luego por el análisis de la elaboración de contenidos pedagógicos ("los saberes que se enseñan"). La metodología utilizada moviliza ciertas herramientas desarrolladas en el seno de la didáctica profesional: el análisis del contexto profesional y de la estructura 
conceptual de la situación. Los resultados expuestos permiten comprender las modalidades de transposición efectuadas a través de la simulación informática y anuncian posibles vías de tratamiento, que serian la continuidad de este trabajo.

Palabras clave

Didáctica profesional, formación universitaria superior, referencias, procesos transpositivos, saberes prácticos, simulador informático

\section{RÉFÉRENCEMENT}

Becerril Ortega, R., Calmettes, B. Fraysse, B., \& Lagarrigue, P. (2009). Des références pour des pratiques de formation. Étude d'une situation de formation technologique supérieure initiale. Activités 6 (1), pp. 29-48, http://www.activites.org/v6n1/v6n1.pdf

Article soumis le 19 février 2008, accepté pour publication le 1er octobre 2008. 